\title{
Influence of dynamic loads on the optimum design of trusses
}

\author{
J. Van Steirteghem ${ }^{1}$, W. Ponsaert ${ }^{1}$, W. P. De Wilde ${ }^{1}$ \\ \& Ph. Samyn ${ }^{1,2}$ \\ ${ }^{1}$ Vrije Universiteit Brussel, Belgium \\ ${ }^{2}$ Samyn and Partners, Belgium
}

\begin{abstract}
The Theory of Morphological Indicators allows a preliminary optimisation of structures at the stage of conceptual design. Samyn and Latteur developed the Indicator of Volume to determine the efficiency of structures at early design stages. The main advantage of this approach is that we only need a very limited number of parameters. Samyn establishes efficiency curves, with respect to minimum volume of material, for trusses in which he neglects buckling. Latteur establishes efficiency curves in which he accounts for buckling. The displacements are usually checked afterwards to verify if the normative constraints are not exceeded. In this paper we argue that for trusses, loaded dynamically and with large spans, dynamics become the dimensioning criterion. We use the Indicator of the First Natural Frequency to determine the first natural frequency of trusses. We find that for fully stressed trusses this natural frequency is usually near to the excitation frequencies of man induced and wind induced vibrations. Therefore, we need to include dynamics in the optimisation procedure. We show that for trusses with important spans very large stress reductions are necessary to obtain acceptable natural frequencies. This stress reduction comes at the cost of a very important and unacceptable increase of the volume of material. We determine which typology (Warren, Howe, Pratt) is the most efficient (minimal volume) with respect to dynamics. Moreover we show that when dynamics is the dimensioning criterion, the influence of buckling on the optimum design is negligible since an important stress reduction is necessary. Finally we propose a work scheme that allows considering dynamics in the Theory of Morphological Indicators and we provide an example.
\end{abstract}

Keywords: conceptual design, morphological indicators, trusses, dynamics. 


\section{Introduction to the theory of morphological indicators}

Morphological Indicators are design tools allowing the optimisation of structures for a chosen criterion (volume, stiffness) at the stage of conceptual design using a limited number of parameters [1]. The Indicator of Volume $W=\sigma V / F L$ allows the comparison of the volume of material used of different structural systems subjected to the same load case. The Displacement Indicator $\Delta=E \delta / \sigma L$ compares the displacement of different structural systems for a given stress level.

Samyn [1] and Latteur [2] established the analytical expressions of both $W$ and $\Delta$ for trusses, beams, arches, cables, cable stayed structures, masts and frames subjected to a limited number of (simple) load cases and support conditions.

For statically determined structures these Morphological Indicators are only function of the geometrical slenderness $L / H$ if instabilities, self weight and second order effect are neglected. Latteur [2] introduced the Indicator of Buckling $\Psi=\mu \sigma L / \sqrt{q E F}$ to take into account the additional volume of material due to buckling. $\Psi$ is a 'global' measure of the sensitivity of a structure to buckling as a function of the material used. Herein, $\mu$ is a reduction factor that determines the effective buckling length of the compression member, $q=I / \Omega^{2}$ is a form factor (with $I$ the moment of inertia and $\Omega$ the section area) that determines the disposition of the material with respect to the center of gravity of the compression member (buckling efficient sections have large value of $q$ ).

Furthermore Samyn [1] and Latteur [2] introduced the Indicator of Self Weight $\Phi=\rho L / \sigma$ to take into account the influence of self weight on the total volume of material. Herein, $\rho$ is the specific weight of the material used.

In $[3,4]$ the authors show that dynamics can become the dimensioning criterion for structures with large spans and/or small stiffness/strength ratio. Therefore, an Indicator of the First Natural Frequency $\Theta=1 / \sqrt{\Delta}$ is introduced $[1,3,4]$.

\section{Indicator of the First Natural Frequency}

\subsection{Classification of loads}

For a structure spanning a length $L$, the total resultant of the loads $F$ consists of:

- The external live loads $F_{l}$

- The external permanent loads $F_{p}$

- The self weight $F_{0}$ 
The external live loads are subdivided in non co-vibrating live loads $F_{l 1}=c F_{l}$ and co-vibrating live loads $F_{l 2}=(1-c) F_{l}$, with $c$ the share of the non covibrating live loads in the total live loads.

Furthermore, we define the following combinations:

- $\quad F_{P}=F_{0}+F_{p}$, the sum of all permanent loads

- $F_{E}=F_{p}+F_{l}$, the sum of all external loads

We can now express the resultant of all loads as follows:

$$
F=F_{0}+F_{E}=F_{P}+F_{l}
$$

When calculating the first natural frequency of a structure, we take into account the co-vibrating loads as vibrating mass. We express the total co-vibrating load $F_{D}$ as follows:

$$
F_{D}=F_{0}+F_{p}+F_{l 2}=F_{P}+(1-c) F_{l}
$$

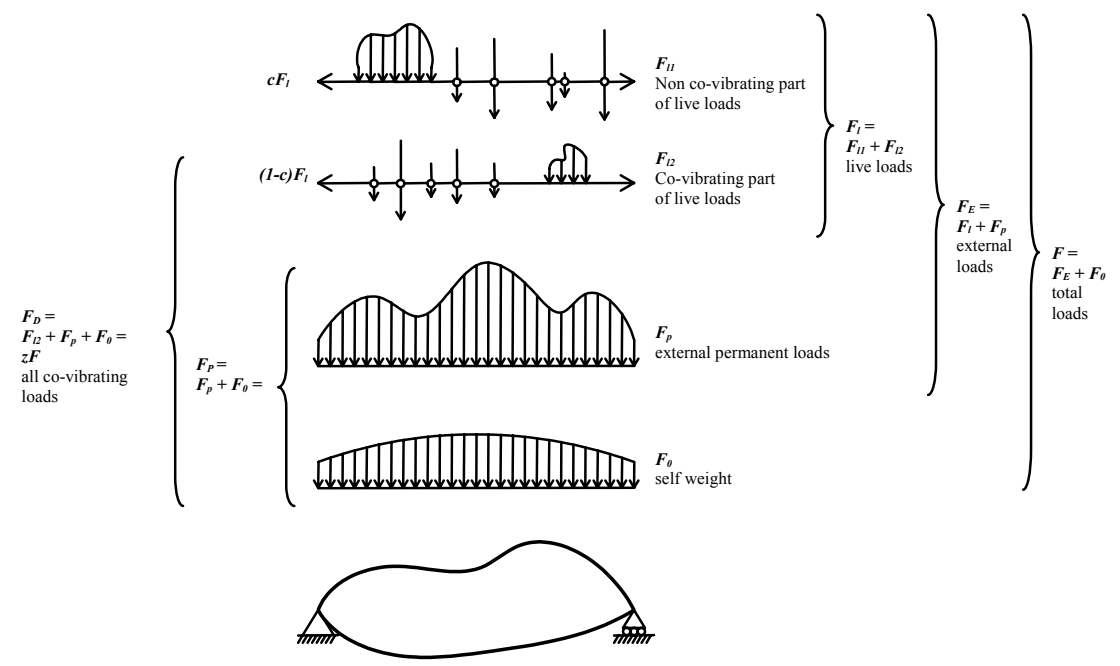

These loads refer to the Ultimate Limit State (ULS) calculation of the structure. For a dynamic analysis, we must consider loads in a Serviceability Limit State (SLS). Hence, we can express $F_{D}$ in SLS

$$
F_{D}^{*}=\frac{F_{P}}{1.35}+(1-c) \frac{F_{l}}{1.50}
$$

And we express the vibrating mass $m_{D}^{*}$ of a structure as:

$$
m_{D}^{*}=\frac{F_{D}^{*}}{g}
$$


Finally, we express the total mass $m$ of a structure as:

$$
m=\frac{F}{g}
$$

\subsection{The first natural frequency of a SDOF system}

By approximating the structure by an undamped single degree of freedom (SDOF) structure, with $m_{D}^{*}$ the vibrating mass in SLS of the structure and $k$ the static stiffness of the structure, the first natural frequency can be expressed as:

$$
f_{1}=\frac{c_{c o r}}{2 \pi} \sqrt{\frac{k}{m_{D}^{*}}}
$$

By reducing a continuous system to a SDOF system, some approximations are made. $c_{c o r}$ is a correction factor that counters these approximations. For beams, it can be determined analytically; for trusses on the other hand, it must be determined numerically.

We can express the vibrating mass as a share of the total mass, with $z^{*}$ the ratio of the co-vibrating load $F_{D}^{*}$ in SLS and the total load $F$ in ULS:

$$
m_{D}^{*}=z^{*} m
$$

By substituting eqns (7) and (5) in eqn (6), the expression of the first natural frequency becomes:

$$
f_{1}=\frac{c_{c o r}}{2 \pi} \sqrt{\frac{g k}{z^{*} F}}
$$

With $\delta=F / k$ the static displacement, eqn (8) becomes:

$$
f_{1}=\frac{c_{c o r}}{2 \pi} \sqrt{\frac{g}{z^{*} \delta}}
$$

or as a function of the Displacement Indicator:

$$
f_{1}=\frac{c_{c o r}}{2 \pi} \sqrt{\frac{g E}{z^{*} \beta \sigma \Delta L}}
$$

The parameter $\beta$ indicates the stress level of the structure.

The Indicator of the First Natural Frequency becomes:

$$
\Theta=\frac{1}{\sqrt{\Delta}}=\frac{2 \pi f_{1}}{c_{c o r}} \sqrt{\frac{z^{*} \beta \sigma L}{g E}}
$$

When we calculate the natural frequency we need a larger number of parameters

- $\quad$ the $\operatorname{span}(L)$

- the material $(\sigma, E)$

- the stress level $(\beta)$

- the ratio of co-vibrating load in SLS and total load in $\operatorname{ULS}\left(z^{*}\right)$. 


\subsection{Determination of the correction factor $c_{c o r}$}

An approximate method to calculate the first natural frequency of a structure, yielding very good results, is Rayleigh's energy method [5]. By using this method we derive the first natural frequency by equating the maximum potential energy to the maximum kinetic energy. For a structure with concentrated loads the fundamental frequency becomes, with $\delta_{j}$ the static displacement under the concentrated load $F_{j}$ :

$$
f=\frac{1}{2 \pi} \sqrt{g \frac{\sum_{j} F_{j} \delta_{j}}{\sum_{j} F_{j} \delta_{j}^{2}}}
$$

We checked the results of this method numerically with Robobat. The results obtained using eqn. (12) underestimate the numerical ones marginally (maximum $10 \%$ for small slendernesses).

\subsection{Indicator of First Natural Frequency}

We can use the Indicator of the First Natural Frequency to determine the first natural frequency of structures.

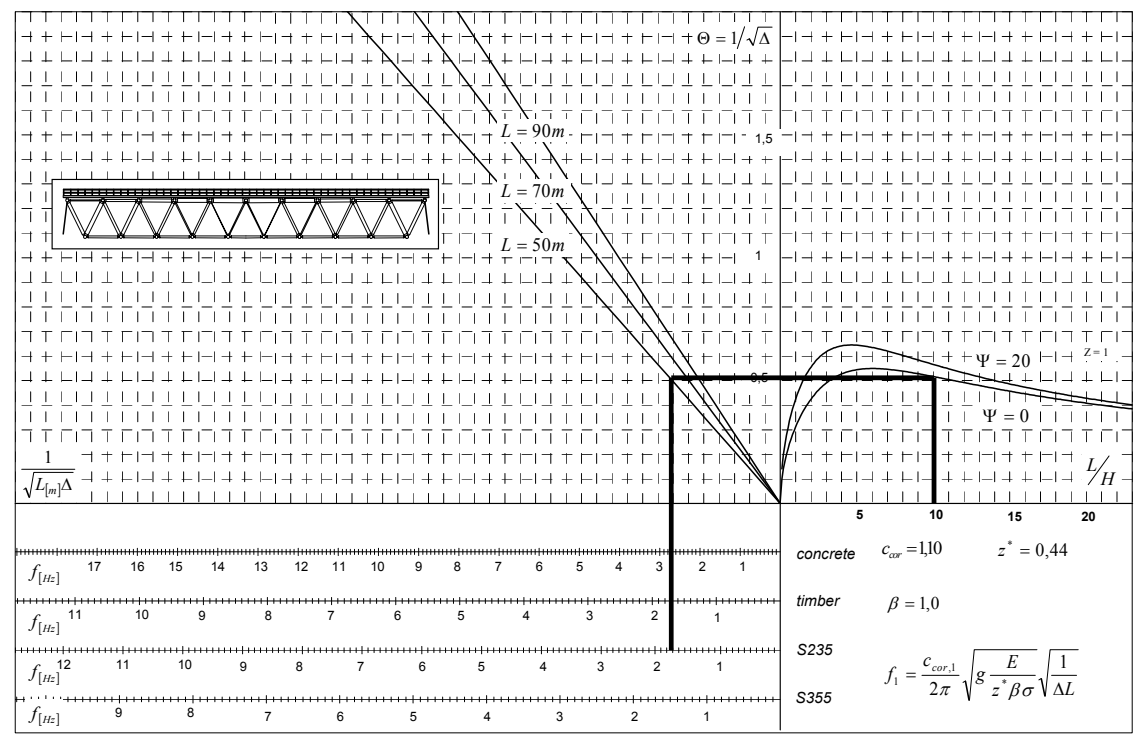

Figure 1: Graph allowing the determination of the first natural frequency of Warren truss with 10 panels. 
In the first quadrant we plot the Indicator of the First Natural Frequency as a function of the overall slenderness. In the second quadrant we plot $1 / \sqrt{\Delta L}$ as a function of $\Theta=1 / \sqrt{\Delta}$. Finally in the third quadrant we plot the frequency abscissa for different material, stress level and value of $z^{*}$. Since we include the material, stress level and value of $z^{*}$ in the frequency abscissa the first and second quadrant must never be changed for this structure.

In this example we determine the first natural frequency of a steel Warren truss with 10 panels and a span of 50m in which we can neglect buckling $(\Psi=0)$. For this example we obtain a first natural frequency of $1,7 \mathrm{~Hz}$.

\section{Trusses dynamically loaded}

\subsection{Scope}

We can now study the behavior of trusses that are subjected to dynamic loads. We consider three truss typologies (Warren, Pratt and Howe):
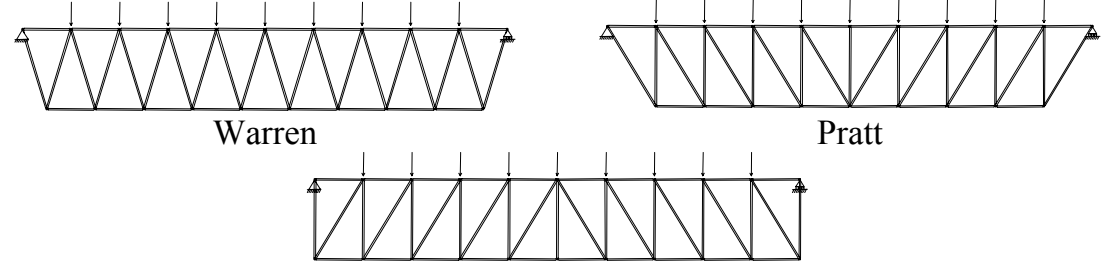

Howe

We consider three truss typologies (Warren, Pratt and Howe) with:

- $\quad$ Three different spans $(\mathrm{L}=20 \mathrm{~m}-50 \mathrm{~m}-70 \mathrm{~m})$

- Three number of panels $(n=4-8-12)$

- Three values of $z^{*}\left(z^{*}=0,15-0,44-0,74\right)$

- Two different materials (timber - steel).

Moreover, we impose that the natural frequency of the structure must be larger than $5 \mathrm{~Hz}$ because the man and wind induced frequencies are usually between $\mathrm{OHz}$ and $5 \mathrm{~Hz}$.

For trusses in which we neglect buckling we can express the constraint on the first natural frequency as a constraint on geometrical slenderness. First, we use eqn. (10) compute an upper bound on $\Delta$ :

$$
0-5 H z \Rightarrow \Delta<\left(\frac{c_{c o r}}{2 \pi \times 5}\right)^{2} \frac{g E}{z^{*} \beta \sigma L}=\Delta_{d y n}=\Delta_{\max }
$$

In [1] we find the expression of the Indicator of Volume of Warren trusses (eqn. (14)) and Howe/Pratt (eqn. (15)) trusses: 


$$
\begin{aligned}
& \frac{\Delta_{d y n}-\sqrt{\Delta_{d y n}^{2}-(n+2)}}{(n+2 / 2 n)}<\frac{L}{H}<\frac{\Delta_{d y n}+\sqrt{\Delta_{d y n}^{2}-(n+2)}}{(n+2 / 2 n)} \\
& \frac{\Delta_{d y n}-\sqrt{\Delta_{d y n}^{2}-(n+1)}}{(n+1 / 2 n)}<\frac{L}{H}<\frac{\Delta_{d y n}+\sqrt{\Delta_{d y n}^{2}-(n+1)}}{(n+1 / 2 n)}
\end{aligned}
$$

On the other hand, if we consider buckling through $\Psi$ this interval is calculated numerically.

\subsection{Results and comparison}

When we select a target slenderness of $L / H=10$, the large majority of combinations (typology, $L, n, z^{*}$, material) need a large stress reduction to obtain a first natural frequency larger than $5 \mathrm{~Hz}$. This stress reduction increases when we use:

- Pratt or Howe truss instead of Warren truss

- A large span

- A large number of panels

- A large value of $z^{*}$

Table 1: Howe-Pratt $(\Psi=0)$ : Necessary stress reduction for target slenderness $\mathrm{L} / \mathrm{H}=8$ for different materials, spans, number of panels and $\mathrm{z}^{*}$.

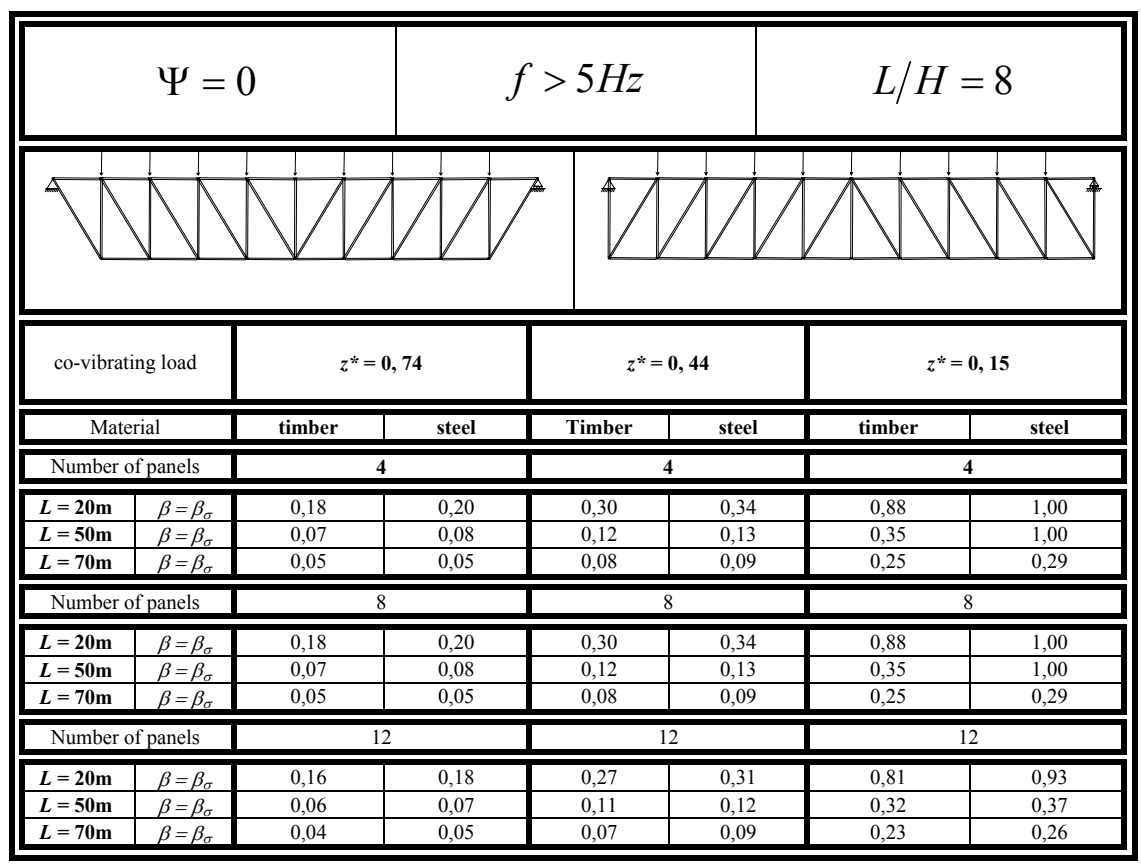


The stiffness/strength ratio of steel and timber is comparable. Table 1 gives an indication of $\beta$, the necessary stress reduction to obtain $f_{1}>5 \mathrm{~Hz}$ for a Howe/Pratt truss.

\section{Conclusions and further research}

\subsection{Conclusions}

In this paper, we showed that we can include dynamics into the Theory of Morphological Indicators. This comes at the cost of a larger number of parameters to consider. However, we believe that in order to be useful we must include the dynamic behaviour of (lightweight) structures. Moreover, we argue that the number of parameters to consider are still small and are all available at the stage of conceptual design.

We developed the Indicator of the First Natural Frequency, $\Theta$, to compute the first natural frequency of structures. The expression of $\Theta$ clearly shows that the problem is not scale independent anymore.

We found that trusses with large spans, large values of $z^{*}$ and small stiffness/strength ratio are sensitive to dynamic loads and need important stress reduction to obtain an acceptable natural frequency.

\subsection{Further research}

In order to make the Theory of Morphological Indicators more powerful in terms of dynamic behaviour, the following topics can be worked out in the future:

- Stress reduction due to fatigue

- Higher natural modes and frequencies

- External damping devices (passif, actif, hybrid)

\section{References}

[1] Samyn, Ph., Etude comparée du volume et du déplacement de structures bidimensionnelles, sous charges verticales entre deux appuis - vers un outil d'évaluation et de pré-dimensionnement des structures, $\mathrm{PhD}$ thesis, Université de Liège, 1999

[2] Latteur, P., Optimisation des treillis, arcs, poutres et câbles sur base d'indicateurs morphologiques - Application aux structures soumises en partie ou en totalité au flambement, $\mathrm{PhD}$ thesis, Vrije Universiteit Brussel, 2000

[3] Van Steirteghem, J., A contribution to the Optimisation of Structures Using Morphological Indicators: (In) Stability and Dynamics, $\mathrm{PhD}$ thesis, Vrije Universiteit Brussel, 2006

[4] Ponsaert, W., Het gebruik van trillingsdempers in balkconstructies, Master thesis, Vriie Universiteit Brussel, 2005

[5] Fertis, D.G., Mechanical and Structural Vibrations, John Wiley \& Sons, New York, 1995 